\title{
Matching information from two independent informative samples
}

\author{
Daniela Marella*, Danny Pfeffermann** 1 \\ *Dipartimento di Scienze della Formazione, Università "Roma Tre" \\ ** Central Bureau of Statistics and Hebrew University of Jerusalem, Israel; \\ University of Southampton, UK.
}

\begin{abstract}
Abstract: Statistical matching deals with the problem of how to combine information collected in different samples taken from the same target population, but on partly different survey variables. The purpose of this paper is to analyze the statistical matching problem under informative sampling designs, when applying the sample likelihood approach. First, a conditional independence assumption is made, which allows to define an identifiable population model under which the conditions guaranteeing the identifiability and estimability of the sample likelihood are investigated. Next, the uncertainty in statistical matching under informative sampling designs is discussed, with particular attention to the three-variate normal case. A simulation experiment illustrating the theoretical results is performed.
\end{abstract}

Key words: conditional independence, informative sampling, matching uncertainty, sample distribution, sample likelihood.

\section{Introduction}

The statistical matching problem consists of combining information collected from different samples drawn from the same population, with only partial overlap between the two samples. Formally, the problem can be described as follows. Let $A$ and $B$ be two independent samples of sizes $n_{A}$ and $n_{B}$ respectively, selected from a population of

\footnotetext{
${ }^{1}$ email: daniela.marella@uniroma3.it,D.Pfeffermann@soton.ac.uk Corresponding author: Daniela Marella - Dipartimento di Scienze della Formazione, Università "Roma Tre" daniela.marella@uniroma3.it
} 
independent records $\left\{\left(x_{i}, y_{i}, z_{i}\right)\right\}$, generated from some joint density $f(x, y, z ; \theta)$, governed by a vector parameter $\theta$. The problem of statistical matching is that $(X, Y, Z)$ are not jointly observed in the two samples. Specifically, suppose that only $(X, Y)$ are observed for the units in sample $A$, and only $(X, Z)$ are observed for the units in sample $B$. See, e.g., [17] and [6]. We assume that the samples $A$ and $B$ have no units in common, which will generally be the case if the population is sufficiently large and the selection probabilities are small.

The goal of statistical matching is to reconstruct a matched (fused) data set in which each record includes measurements on $(X, Y, Z)$, which users may treat as a "completely" observed data set from a single source. Due to the absence of joint observations of $Z$ and $Y$ for given $X$, the distribution $f(x, y, z ; \theta)$ is generally not identifiable. In order to overcome this problem, two approaches have been considered in the literature. The first approach assumes conditional independence between $Y$ and $Z$ given $X$ (hereafter CIA); see, e.g., [11]. The CIA has a very important role in statistical matching, the reason being that under the CIA, the distribution of $(X, Y, Z)$ is identifiable and directly estimable from the information provided by the two samples. Appropriateness of the CIA is discussed in several papers. We cite, among others, [22] and [19]. The second approach assumes the existence of external auxiliary information regarding the statistical relationship between $Y$ and $Z$; see, e.g., [23]. However, commonly, neither approach is applicable. The CIA is rarely met in practice and relevant external auxiliary information is not often available. The lack of joint observations on the variables of interest implies uncertainty about the model holding for $(X, Y, Z)$. In other words, the sample information provided by $A$ and $B$ is not sufficient to enable to distinguish among plausible models for $(X, Y, Z)$, resulting in identification problems. In a parametric setting, the consequence of the identification problem is that only ranges of plausible values of the missing records, obtained from models fitted to the available sample information can be defined. Intervals defined by 
these ranges are known in the literature as uncertainty intervals. References tackling the problem of assessing the statistical matching uncertainty in the context of independent and identically observations $($ i.i.d) are [8], [20], [10] and [17]. Uncertainty in statistical matching in a nonparametric setting under the i.i.d assumption is considered in [1], [2] and $[3]$.

In practice, the i.i.d assumption is itself questionable, particularly when dealing with sample survey data. The sample selection in survey sampling involves complex sampling designs based on different levels of clustering and differential inclusion probabilities, which could be correlated with the survey variables of interest. This can violate the independence assumption and result in different distributions of the observed data from the distribution holding in the population from which the samples are drawn. See Section 3. Statistical matching in complex sample surveys is studied in [20], [18] and [4]. [20] proposes to compute new sampling weights for all the units in $A \cup B$. However, this approach is seldom applied since it requires to assess the inclusion probabilities of the units in one sample under the sampling design of the other sample. [18] proposes to calibrate the sampling weights in $A$ and $B$ such that the new weights, when applied to the measured $x$-values in the two samples, reproduce the known (estimated) population totals of $X$. Next, the author estimates the joint distribution of categorical variables $Y$ and $Z$ under the CIA. [4] deals with the statistical matching problem for complex sample surveys non-parametrically. The authors propose to estimate the distribution function of variables which are not jointly observed based on an iterative proportional fitting algorithm, and show how to evaluate its reliability.

The aim of the present paper is to analyze the statistical matching problem for the case where the sampling processes used to select the samples $A$ and $B$ are informative for the target variables of interest. As already mentioned, official survey data are usually collected from samples drawn by probability sampling. When the inclusion probabilities 
are related to the value of the target outcome variable even after conditioning on the model covariates, the observed outcomes are no longer representative of the population outcomes and the model holding for the sample data is then different from the model holding in the population. This, quite common phenomenon, is known in the survey sampling literature as informative sampling. In this case, conventional analysis, which ignores the informative sampling effects may yield large bias and erroneous inference, as illustrated, for example, in the book edited by [24]. See [14] for discussion of the notion of informative sampling and review of methods to deal with this problem. Returning to statistical matching, knowledge of the sampling designs underlying the selection of the samples $A$ and $B$ and accounting for them, is crucial for successful matching. This is true even under the simplified CIA framework.

The paper is organized as follows. In Section 2 we summarize briefly the parametric solution to the matching problem under the CIA, for the case where the sampling process is noninformative. Section 3 considers the case of informative sampling under the CIA and defines the corresponding sample likelihood for the statistical matching problem. The use of the sample likelihood enables estimating the corresponding population distributions and to impute the missing values. The conditions under which the sample models are identifiable and estimable from the information provided by the samples $A$ and $B$ are investigated in Section 4. Section 5 analyses the case of a three-variate normal distribution, investigating the effects of different informative sampling designs on the population model. In Section 6 the CIA assumption is relaxed, and the uncertainty in statistical matching under informative sampling is restudied, illustrated in Section 7, where we again restrict to the three-variate normal case. Section 8 contains results of simulation experiments used to illustrate the theoretical results. We conclude with a brief summary and suggestions for further research in Section 9. All proofs and additional results are deferred to the Supplementary Material. 


\section{Statistical matching under CIA and noninformative sampling}

Suppose that the population values $D_{p}=\left\{\left(x_{1}, y_{1}, z_{1}\right), \ldots,\left(x_{N}, y_{N}, z_{N}\right)\right\}$ are independent realizations from some joint probability density function $(p d f) f(x, y, z ; \theta)$, indexed by a vector parameter $\theta$. When the CIA holds, the population model $f_{p}(x, y, z ; \theta)$ can be factorized as

$$
f_{p}\left(x_{i}, y_{i}, z_{i} ; \theta\right)=f_{p}\left(x_{i} ; \theta_{X}\right) f_{p}\left(y_{i} \mid x_{i} ; \theta_{Y \mid X}\right) f_{p}\left(z_{i} \mid x_{i} ; \theta_{Z \mid X}\right)
$$

where the (vector) parameters $\theta_{X}, \theta_{Y \mid X}$ and $\theta_{Z \mid X}$ governing the corresponding three distributions, are assumed to be distinct. Under noninformative sampling, such that the population model holds also for the sample data, the model (2.1) is identifiable and directly estimable from the two distinct samples $A$ and $B$. Furthermore, when the distributions in (2.1) are parametric and the parameters $\theta_{X}, \theta_{Y \mid X}, \theta_{Z \mid X}$ are distinct, it is possible to resort to maximum likelihood estimation (MLE), the MLE of $\theta=$ $\left(\theta_{X}, \theta_{Y \mid X}, \theta_{Z \mid X}\right)$ can be obtained by considering separately the corresponding three likelihoods. Once the parametric models have been estimated, a matched data set can be obtained by imputing the missing values in the combined file $A \cup B$ from the distributions of the corresponding missing target variables, given the observed variables.

\section{The sample likelihood under CIA and informative sampling}

Let $V_{p}^{A}, V_{p}^{B}$ be sets of population values of design variables used for selecting the samples $A$ and $B$, respectively. The design variables often contain strata and cluster indicators, and/or variables of measures of size, used for probability proportional to size sampling. The design values $V_{p}^{A}, V_{p}^{B}$ are known to the persons selecting the samples, but generally not to analysts analysing the data. Some or all of the variables $(X, Y, Z)$ may be included among the design variables, but at least $Y$ and $Z$ are only known to the analyst of the sample data. We assume that the population data, $D_{p}$ and the values of $V_{p}^{A}, V_{p}^{B}$ are realizations of random processes, implying that the first order sample 
inclusion probabilities $\pi_{i}^{A}=\operatorname{Pr}(i \in A), \pi_{i}^{B}=\operatorname{Pr}(i \in B)$ may be viewed as random as well. The sample inclusion probabilities contain invaluable information about the relationship between the distribution of the sample data and the distribution in the population from which the samples are taken. Denote by $w_{i}^{A}=1 / \pi_{i}^{A}, w_{i}^{B}=1 / \pi_{i}^{B}$ the (basic) sampling weights for the two samples. The sample data may be viewed therefore as the outcome of two random processes: the first process generates the values for the $N$ population units, while the second process selects a sample from the finite population according to the underlying sampling design. As it is often the case in a secondary analysis, the data available to the analyst consist of only the two samples $A=\left\{\left(x_{a}, y_{a}, w_{a}^{A}\right)\right\}$ and $B=\left\{\left(x_{b}, z_{b}, w_{b}^{B}\right)\right\}$ of sizes $n_{A}$ and $n_{B}$, respectively. Let $I_{a}^{A}$ be the sample $A$ indicator; $I_{a}^{A}=1$ if population unit $a \in A, I_{a}^{A}=0$ otherwise. If $Z$ was observed in $A$, then following [12], the marginal sample $p d f$ of $\left(x_{a}, y_{a}, z_{a}\right)$ for $a \in A$ is defined as

$$
\begin{aligned}
f_{A}\left(x_{a}, y_{a}, z_{a}\right) & =\frac{P\left(I_{a}^{A}=1 \mid x_{a}, y_{a}, z_{a}\right)}{P\left(I_{a}^{A}=1\right)} f_{p}\left(x_{a}, y_{a}, z_{a}\right) \\
& =f_{A}\left(x_{a}\right) f_{A}\left(y_{a} \mid x_{a}\right) f_{A}\left(z_{a} \mid x_{a}, y_{a}\right)
\end{aligned}
$$

and under independence between observations corresponding to different sampling units, (see Remark 1 below), the corresponding sample likelihood based on the sample $A$ (without parameter notation, see below) is,

$$
L^{A}=\prod_{a=1}^{n_{A}} f_{A}\left(x_{a}, y_{a}, z_{a}\right)=\prod_{a=1}^{n_{A}} f_{A}\left(x_{a}, y_{a}\right) f_{A}\left(z_{a} \mid x_{a}, y_{a}\right)
$$

However, since the variable $Z$ is not observed in $A$, the sample likelihood of $\theta$ based on the observed data in $A$ is obtained by integrating the missing data out of the complete 
sample likelihood (3.2). The observed sample likelihood of $A$ is thus given by,

$$
L_{O b s}^{A}\left(\theta_{X}, \theta_{Y \mid X}, \gamma^{A}\right)=\int \prod_{a=1}^{n_{A}} f_{A}\left(x_{a}, y_{a}, z_{a}\right) d z_{a}=\prod_{a=1}^{n_{A}} f_{A}\left(x_{a}, y_{a} ; \theta_{X}, \theta_{Y \mid X}, \gamma^{A}\right)
$$

where $\gamma^{A}$ represents any additional parameters defining the sample distribution, resulting from the sample process. See Equations (3.6) and (3.7) below.

Remark 1. [12] establish general conditions under which for independent observations under the population model, the sample measurements are asymptotically independent under the sample model, when increasing the population size but holding the sample size fixed. This permits approximating the sample likelihood by the product of the sample pdfs over the corresponding sample observations. In Section 4 we discuss the identifiability and estimability of the sample pdf.

Similarly, the observed sample likelihood of $B$ is,

$$
L_{O b s}^{B}\left(\theta_{X}, \theta_{Z \mid X}, \gamma^{B}\right)=\int \prod_{b=1}^{n_{B}} f_{B}\left(x_{b}, y_{b}, z_{b}\right) d y_{b}=\prod_{b=1}^{n_{B}} f_{B}\left(x_{b}, z_{b} ; \theta_{X}, \theta_{Z \mid X}, \gamma^{B}\right) .
$$

Hence, the sample likelihood of the sample $A \cup B$ is,

$$
\begin{aligned}
L_{O b s}^{A \cup B}\left(\theta, \gamma^{A}, \gamma^{B}\right)= & \prod_{a=1}^{n_{A}} f_{A}\left(x_{a}, y_{a} ; \theta_{X}, \theta_{Y \mid X}, \gamma^{A}\right) \prod_{b=1}^{n_{B}} f_{B}\left(x_{b}, z_{b} ; \theta_{X}, \theta_{Z \mid X}, \gamma^{B}\right) \\
= & \prod_{a=1}^{n_{A}} f_{A}\left(y_{a} \mid x_{a} ; \theta_{Y \mid X}, \gamma^{A}\right) \prod_{b=1}^{n_{B}} f_{B}\left(z_{b} \mid x_{b} ; \theta_{Z \mid X}, \gamma^{B}\right) \\
& \prod_{a=1}^{n_{A}} f_{A}\left(x_{a} ; \theta_{X}, \gamma^{A}\right) \prod_{b=1}^{n_{B}} f_{B}\left(x_{b} ; \theta_{X}, \gamma^{B}\right),
\end{aligned}
$$

where the parameters $\theta_{X}, \theta_{Y \mid X}, \theta_{Z \mid X}$, governing the population $p d f \mathrm{~s}$, are orthogonal because of the conditional independence of $Y$ and $Z$ given $X$. Notice that (3.5) has a similar structure to the observed data likelihood under noninformative sampling, as defined in [17], the big difference being that the population models $f_{p}\left(x_{a}, y_{a}\right), f_{p}\left(x_{b}, z_{b}\right)$ 
are replaced in (3.5) by the sample models $f_{A}\left(x_{a}, y_{a}\right), f_{B}\left(x_{b}, z_{b}\right)$. The maximum likelihood estimates are obtained by maximizing separately the corresponding three sample likelihoods in the right hand side of (3.5), where the estimator of $\theta_{X}$ is obtained by maximizing $\prod_{a=1}^{n_{A}} f_{A}\left(x_{a}\right) \prod_{b=1}^{n_{B}} f_{B}\left(x_{b}\right)$, thus utilizing the data in both samples. Following [12] and [13], the sample $p d f$ s featuring in (3.5) can be expressed alternatively as,

$$
\begin{aligned}
f_{A}\left(x_{a} ; \theta_{X}, \gamma^{A}\right) & =\frac{P\left(I_{a}^{A}=1 \mid x_{a} ; \gamma^{A}\right)}{P\left(I_{a}^{A}=1 ; \theta_{X}, \gamma^{A}\right)} f_{p}\left(x_{a} ; \theta_{X}\right)=\frac{E_{p}\left(\pi_{a}^{A} \mid x_{a} ; \gamma^{A}\right)}{E_{p}\left(\pi_{a}^{A} ; \theta_{X}, \gamma^{A}\right)} f_{p}\left(x_{a} ; \theta_{X}\right) \\
& =\frac{E_{A}\left(w_{a}^{A} ; \theta_{X}, \gamma^{A}\right)}{E_{A}\left(w_{a}^{A} \mid x_{a} ; \gamma^{A}\right)} f_{p}\left(x_{a} ; \theta_{X}\right)
\end{aligned}
$$

where $f_{p}\left(x_{a} ; \theta_{X}\right)$ represents the corresponding population $p d f$. Equation (3.6) uses the relationship between the population $p d f$ and the sample $p d f$. Notice that the expectations in the left- and right hand side of this relationship refer to different distributions. The relationship $E_{p}\left(\pi_{a}^{A} \mid x_{a} ; \gamma^{a}\right)=1 / E_{A}\left(w_{a}^{A} \mid x_{a} ; \gamma^{A}\right)$ has been established in [13], where $E_{p}, E_{A}$ denote expectations under the population and sample distributions, respectively. When $P\left(I_{a}^{A}=1 \mid x_{a} ; \gamma^{A}\right)=P\left(I_{a}^{A}=1 ; \theta_{X}, \gamma^{A}\right)$ for each $x_{a}$, the population and sample models are the same and the sampling design may be ignored for inference on the parameters $\theta_{X}$. The conditional marginal sample $p d f f_{A}\left(y_{a} \mid x_{a} ; \theta_{Y \mid X}, \gamma^{A}\right)$ is defined as the conditional $p d f$ of $y_{a} \mid x_{a}$ given that unit $a$ is in the sample $A$. Similarly to (3.6),

$$
f_{A}\left(y_{a} \mid x_{a} ; \theta_{Y \mid X}, \gamma^{A}\right)=\frac{E_{A}\left(w_{a}^{A} \mid x_{a} ; \theta_{Y \mid X}, \gamma^{A}\right) f_{p}\left(y_{a} \mid x_{a} ; \theta_{Y \mid X}\right)}{E_{A}\left(w_{a}^{A} \mid x_{a}, y_{a} ; \gamma^{A}\right)}
$$

Similar expressions to (3.6) and (3.7) are obtained for the sample $p d f_{\mathrm{s}} f_{B}\left(x_{b}\right)$ and $f_{B}\left(z_{b} \mid x_{b}\right)$ operating in the sample $B$. The sample distributions are functions of the corresponding population $p d f \mathrm{~s}$ and the respective conditional expectations of the sampling weights. The parameters $\theta_{X}, \theta_{Y \mid X}, \theta_{Z \mid X}$ governing the three population models can be estimated from the corresponding sample data, by MLE. Furthermore, the expectations displayed in the sample $p d f \mathrm{~s}$ can be estimated from the observed data, 
using classical model fitting procedures. Fixing the unknown parameters featuring in these expectations at their estimated values allows to maximize the resulting likelihoods only with respect to the parameters indexing the population $p d f \mathrm{~s}$, thus simplifying and stabilizing the maximization process. For example, the expectation $E_{A}\left(w_{a}^{A} \mid x_{a}, y_{a} ; \gamma^{A}\right)$ in (3.7) can be estimated by regressing $w_{a}^{A}$ against $\left(x_{a}, y_{a}\right)$, using the observations $\left\{\left(x_{a}, y_{a}, w_{a}^{A}\right), a \in A\right\}$. See, e.g., [14] and [15] for examples of regression models that can be used for this purpose, depending on the problem at hand. Alternatively, the expectation $E_{A}\left(w_{a}^{A} \mid x_{a}, y_{a} ; \gamma^{A}\right)$ can be estimated non-parametrically, as in [7]. Once the parameters governing the population model have been estimated, a fused dataset $D_{f}=\left\{\left(\tilde{x}_{i}, \tilde{y}_{i}, \tilde{z}_{i}\right), i=1, \ldots, \tilde{n}\right\}$, where $\tilde{n}$ is the desired sample size, (possibly $\tilde{n}=N$, the population size), can be constructed, such that each record includes measurements of $(X, Y, Z)$, which users may treat as a "completely" observed dataset, with a similar distribution to the population distribution. Formally, one may employ the following procedure: (i) Generate $\tilde{n}$ observations $\tilde{x}_{i}$ from $f_{p}\left(x_{i} ; \widehat{\theta}_{X}\right)$; (ii) For each $i=1, \ldots, \tilde{n}$ draw at random a value $\tilde{y}_{i}$ from $f_{p}\left(y_{i} \mid x_{i} ; \widehat{\theta}_{Y \mid X}\right)$ (iii) For each $i=1, \ldots, \tilde{n}$ draw at random a value $\tilde{z}_{i}$ from $f_{p}\left(z_{i} \mid x_{i} ; \widehat{\theta}_{Z \mid X}\right)$.

Under general likelihood theory, the population model parameter estimators are consistent, guaranteeing that for sufficiently large samples $A$ and $B$, the synthetic dataset $D_{f}$ defines a genuine sample from the population $p d f f_{p}\left(x_{i}, y_{i}, z_{i} ; \theta\right)$.

Remark 2. Unlike in the case of noninformative sampling, it is generally not correct to only impute the missing z-values for the sample A, and the missing y-values for the sample $B$, and consider the union of the two samples as the final matched data set of size $n_{A}+n_{B}$. The reason for this is that the sample $A \cup B$ is informative and does not represent the population distribution adequately. Thus, although the z-values can be generated from the population model $f_{p}\left(z_{i} \mid x_{i} ; \widehat{\theta}_{Z \mid X}\right)$, the observed $x$-and $y$-values may not represent the corresponding population $x$ - and $y$-values adequately. 


\section{Identifiability and estimability of the sample model}

As outlined in Section 3, we base the inference on the unknown model parameters on the sample likelihood, which requires that the corresponding sample $p d f_{\mathrm{s}} f_{A}\left(x_{a}, y_{a}\right)$ and $f_{B}\left(x_{b}, z_{b}\right)$ are identifiable and estimable from the available sample information. The sample model $f_{A}\left(x_{a}, y_{a}\right)$ is identifiable, if no different pairs $\left[f_{p}^{1}\left(x_{a}, y_{a}\right), P^{1}\left(I_{a}^{A}=1 \mid x_{a}, y_{a}\right)\right]$, $\left[f_{p}^{2}\left(x_{a}, y_{a}\right), P^{2}\left(I_{a}^{A}=1 \mid x_{a}, y_{a}\right)\right]$ exist, which induce the same sample $p d f f_{A}\left(x_{a}, y_{a}\right)$. See [16] and references therein for conditions guaranteeing the identifiability of the sample model. Similar conditions are required for the identifiability of the sample model $f_{B}\left(x_{b}, z_{b}\right)$. By estimability we mean that the population and sampling parameters appearing in the sample models can be estimated from the available data when using the sample likelihood for estimation. As stated in Proposition 1 below, the estimability of the vector parameter $\theta=\left(\theta_{X}, \theta_{Y \mid X}, \theta_{Z \mid X}\right)$ based on the likelihood (3.5) depends on both the fulfillment of the CIA at the sample level and the properties of the sampling process.

Remark 3. Even if the CIA holds in the population, it does not necessarily hold in the sample because even conditionally on $X$, the sampling process could induce dependence between the not jointly observed variables $Y$ and $Z$. See Example 3 in Section 5.

Proposition 1. If the CIA holds for the population values and $P\left(I_{a}^{A}=1 \mid x_{a}, y_{a}, z_{a}\right)=$ $P\left(I_{a}^{A}=1 \mid x_{a}, y_{a}\right)$ for all $z_{a}$ then,

1. The CIA holds in sample $A$.

2. The sample model $f_{A}\left(x_{a}, y_{a}\right)$ is estimable from the sample information in $A$.

Proof of Proposition 1 is in the Supplementary Material, Section S1.

Remark 4. Under the conditions of Proposition 1, it is possibile to impute the missing Z values in sample $A$ and obtain complete observations from the sample model $f_{A}\left(x_{a}, y_{a}, z_{a}\right)$, by estimating $f_{p}(z \mid x)$ from sample B. However, as already noted in Remark 2, the 
extended sample may be informative and not represent the population distribution adequately.

It is not correct to conclude from Proposition 1 that if the sampling process in $A$ depends on the $z$-values, the sampling and the population parameters featuring in $f_{A}\left(x_{a}, y_{a}\right)$ can not be estimated. The sampling probabilities $\pi_{a}^{A}$ may depend on many unobserved design variables including $Z$ and yet, by definition of the sample $p d f$ $f_{A}\left(x_{a}, y_{a}\right)$, one only needs to model the probability $P\left(I_{a}^{A}=1 \mid x_{a}, y_{a}\right)$. As discussed in Section 3, the latter probability can be estimated from the sample data by regressing the sampling weights against $\left(x_{a}, y_{a}\right)$. Moreover, the resulting sample model (3.7) can be tested, as discussed and illustrated in [15] and [7]. A similar proposition to Proposition 1 applies to the sample $B$.

Remark 5. In the case of noninformative sampling, it is sufficient to assume the CIA for proper inference on the population parameters. However, under informative sampling, one needs also to verify that the sample pdfs featuring in the likelihood (3.5) can be estimated and tested on the basis of the available information for the samples $A$ and $B$.

\section{Informative sampling from a trivariate normal population satisfying the}

\section{CIA}

In this section we consider the case where the population distribution is trivariate normal that satisfies the CIA. In particular, we study the effect of alternative informative sampling designs on the inference process. Suppose that $(X, Y, Z)$ is normal with mean $\mu=\left(\mu_{X}, \mu_{Y}, \mu_{Z}\right)$ and variance-covariance $(\mathrm{V}-\mathrm{C})$ matrix $\Sigma$, such that $\sigma_{Y Z}=\sigma_{Y X} \sigma_{Z X} / \sigma_{X}^{2}$. Under the CIA, the population distribution can be factorized as in (2.1), where the marginal distribution of $\mathrm{X}$ is $N\left(\mu_{X}, \sigma_{X}^{2}\right)$ and the conditional distribution of $\mathrm{Y}$ given $X=x$ is $N\left(\mu_{Y \mid x}, \sigma_{Y \mid X}^{2}\right)$, with $\mu_{Y \mid x}=\beta_{0}+\beta_{1} x, \beta_{0}=\mu_{Y}-\beta_{1} \mu_{X}, \beta_{1}=\sigma_{X Y} / \sigma_{X}^{2}$, $\sigma_{Y \mid X}^{2}=\sigma_{Y}^{2}-\beta_{1}^{2} \sigma_{X}^{2}$. Similar expressions hold for the conditional distribution of $Z$ given $X=x$ with parameters $\left(\alpha_{0}, \alpha_{1}, \sigma_{Z \mid X}^{2}\right)$. In Examples 1, 2 and 4 below, (but not in Example 
3), the CIA is preserved by the sampling process. Furthermore, the sample model and the population model are in the same family and only differ in some or all the parameters. Yet, since the units in $A$ have missing $Z$ values while the units in $B$ have missing $Y$ values, the reference sample model is the sample $p d f f_{A \cup B}^{s m}\left(x_{i}, y_{i}, z_{i}\right)$,

$$
f_{A \cup B}^{s m}\left(x_{i}, y_{i}, z_{i}\right)=f_{A \cup B}\left(x_{i}\right) f_{A}\left(y_{i} \mid x_{i}\right) f_{B}\left(z_{i} \mid x_{i}\right) .
$$

Additionally, in Examples 1, 2 the samples $A$ and $B$ are selected with the same sampling probabilities. Clearly, in real situations the sampling designs giving rise to the two samples might be different as considered in Example 4.

Example 1. Following [12], suppose that the sample inclusion probabilities in $A$ and $B$ have expectations,

$$
E_{p}\left(\pi_{i} \mid x_{i}, y_{i}, z_{i}\right)=\kappa \exp \left\{\gamma_{X} x_{i}+\gamma_{Y} y_{i}+\gamma_{Z} z_{i}\right\}
$$

where $\kappa$ guarantees that the expectation is less or equal to one. With regard to Equation (5.2), under probability proportional to size sampling

$$
E_{p}\left(\pi_{i} \mid x_{i}, y_{i}, z_{i}\right)=N n_{A} \frac{\exp \left\{\gamma_{X} x_{i}+\gamma_{Y} y_{i}+\gamma_{Z} z_{i}\right\}}{N \sum_{j=1}^{N} \exp \left\{\gamma_{X} x_{j}+\gamma_{Y} y_{j}+\gamma_{Z} z_{j}\right\}}
$$

For large $N$ (the common case), $\sum_{j=1}^{N} \exp \left\{\gamma_{X} x_{j}+\gamma_{Y} y_{j}+\gamma_{Z} z_{j}\right\} / N$ can be considered as a constant and so is $\kappa$, in which case it cancels out in the derivation of the sample distribution. See [12] and [13] for discussion and many other examples.

Under (5.2), the joint sample pdf can be factorized as,

$$
f_{S}\left(x_{i}, y_{i}, z_{i}\right)=f_{S}\left(x_{i}\right) f_{S}\left(y_{i} \mid x_{i}\right) f_{S}\left(z_{i} \mid x_{i}\right)
$$

where $S=A, B$ denotes the sample and 
(a) $f_{S}\left(x_{i}\right)$ is $N\left(\mu_{X}+\left(\gamma_{X}+\beta_{1} \gamma_{Y}+\alpha_{1} \gamma_{Z}\right) \sigma_{X}^{2}, \sigma_{X}^{2}\right)$;

(b) $f_{S}\left(y_{i} \mid x_{i}\right)$ is $N\left(\mu_{Y \mid x_{i}}+\gamma_{Y} \sigma_{Y \mid X}^{2}, \sigma_{Y \mid X}^{2}\right)$;

(c) $f_{S}\left(z_{i} \mid x_{i}\right)$ is $N\left(\mu_{Z \mid x_{i}}+\gamma_{Z} \sigma_{Z \mid X}^{2}, \sigma_{Z \mid X}^{2}\right)$;

(d) the sample pdf $f_{A \cup B}^{s m}\left(x_{i}, y_{i}, z_{i}\right)$ (5.1), is $N\left(\mu_{A \cup B}, \Sigma_{A \cup B}\right)$, where the sample $V$-C matrix $\Sigma_{A \cup B}$ is the same as for the population distribution, $\Sigma_{A \cup B}=\Sigma$,

thus showing that the CIA holds for the sample. See Supplementary Material, Section S2.

Example 2. Following [12], suppose that the sample inclusion probabilities in $A$ and $B$ have expectations,

$$
E_{p}\left(\pi_{i} \mid x_{i}, y_{i}, z_{i}\right)=\kappa \exp \left\{\gamma_{X} x_{i}+\gamma_{Y} y_{i}+\gamma_{2 Y} y_{i}^{2}\right\}
$$

with $\gamma_{2 Y}<0$. The joint sample pdf can be factorized in this case as in (5.4) where

(a) $f_{S}\left(x_{i}\right)$ is $N\left(\mu_{X}+\left(\gamma_{X}+\beta_{1} \gamma_{Y}\right) \sigma_{X}^{2}, \sigma_{X}^{2}\right)$;

(b) $f_{S}\left(y_{i} \mid x_{i}\right)$ is $N\left(\frac{\mu_{Y \mid x_{i}}+\gamma_{Y} \sigma_{Y \mid X}^{2}}{1-2 \gamma_{2 Y} \sigma_{Y \mid X}^{2}}, \frac{\sigma_{Y \mid X}^{2}}{1-2 \gamma_{2 Y} \sigma_{Y \mid X}^{2}}\right)$;

(c) $f_{S}\left(z_{i} \mid x_{i}\right)$ is $N\left(\mu_{Z \mid x_{i}}, \sigma_{Z \mid X}^{2}\right)$;

(d) the joint sample pdf $f_{A \cup B}^{s m}\left(x_{i}, y_{i}, z_{i}\right)$ (5.1) is $N\left(\mu_{A \cup B}, \Sigma_{A \cup B}\right)$;

(e) for $\gamma_{2 Y}<0$, the sample correlation matrix $\Phi_{A \cup B}$ is positive semidefinite.

thus showing that the CIA holds for the sample. Note that by Proposition 1, $f_{S}\left(z_{i} \mid x_{i}\right)=$ $f_{p}\left(z_{i} \mid x_{i}\right)$ with $S=A, B$. See Supplementary Material, Section S3.

Example 3. Suppose that the sample inclusion probabilities in $A$ and $B$ have expectations,

$$
E_{p}\left(\pi_{i} \mid x_{i}, y_{i}, z_{i}\right)=\kappa \exp \left\{\gamma_{X} x_{i}+\gamma_{Y Z} y_{i} z_{i}\right\}
$$


In this case, the CIA does not hold for the sample, and the sample pdf is no longer trivariate normal. See Supplementary Material, Section S4.

Example 4. Suppose that the sampling inclusion probabilities in $A$ and $B$ have expectation given by (5.2) and (5.5), respectively;

$$
\begin{aligned}
& E_{p}\left(\pi_{i}^{A} \mid x_{i}, y_{i}, z_{i}\right)=\kappa_{A} \exp \left\{\gamma_{X}^{A} x_{i}+\gamma_{Y}^{A} y_{i}+\gamma_{Z}^{A} z_{i}\right\} \\
& E_{p}\left(\pi_{i}^{B} \mid x_{i}, y_{i}, z_{i}\right)=\kappa_{B} \exp \left\{\gamma_{X}^{B} x_{i}+\gamma_{Y}^{B} y_{i}+\gamma_{2 Y}^{B} y_{i}^{2}\right\}
\end{aligned}
$$

(different expectations in the two samples). The joint sample pdf $f_{A \cup B}^{s m}\left(x_{i}, y_{i}, z_{i}\right)$ (5.1) is $N\left(\mu_{A \cup B}, \Sigma\right)$. See Supplementary Material, Section S5.

\section{Uncertainty analysis under informative sampling}

So far, we assumed that the joint population distribution satisfies the CIA. However, the CIA may not hold in practice and the lack of joint measurements on the variables $(X, Y, Z)$ disallows to distinguish between different plausible distributions that possibly hold for them. Let $H_{p}$ be the set of population $p d f_{\mathrm{s}} f_{p}(x, y, z ; \theta)$ having bivariate marginal $p d f_{\mathrm{s}} f_{p}\left(x, y ; \theta_{X Y}\right)$ and $f_{p}\left(x, z ; \theta_{X Z}\right)$, which we assume to be known except for their underlying parameters.

$$
\begin{array}{r}
H_{p}\left[f_{p}(x, y), f_{p}(x, z)\right]=\left\{f_{p}(x, y, z): \int f_{p}(x, y, z) d z=f_{p}(x, y),\right. \\
\left.\int f_{p}(x, y, z) d y=f_{p}(x, z)\right\} .
\end{array}
$$

Each distribution in $H_{p}$ is viewed as a plausible joint distribution of the variables $(X, Y, Z)$. Following [4], we refer to each such distribution as a matching distribution. The larger the class $H_{p}$, the more uncertain is the model for $(X, Y, Z)$. At the sample level, the matching distribution should be chosen from the class defined by (6.1), but with bivariate $p d f$ s estimated on the basis of the sample data. Yet, the parameters $\theta_{Y Z}$ (part 
of the vector $\theta$ indexing the $\left.p d f f_{p}(x, y, z ; \theta)\right)$ cannot be estimated from the samples $A$ and $B$, implying that instead of point estimates, one can only construct a set of plausible values for $\theta_{Y Z}$, which are consistent with the estimates of $\theta_{X Y}$ and $\theta_{X Z}$. Each plausible estimate of $\theta_{Y Z}$ defines a plausible model in the class $H_{p}$. Note, however, that it is not possible to prioritize one model over another, given the available sample data. As noted before, we assume that the form of the population distribution $f_{p}(x, y, z ; \theta)$ is known so that the uncertainty is with regard to the parameters $\theta_{Y Z}$. Suppose that a value $\theta_{Y Z}^{*}$ is chosen from the set of plausible values of $\theta_{Y Z}$ and $f_{p}\left(x, y, z ; \theta_{Y Z}^{*}\right)=f_{p}^{*}(x, y, z) \in H_{p}$ is chosen as the matching distribution of $(X, Y, Z)$, whereas the true population $p d f$ is $f_{p}(x, y, z) \in H_{p}$. The discrepancy between $f_{p}^{*}(x, y, z)$ and $f_{p}(x, y, z)$ is the matching error. The smaller the matching error, the closer is the $p d f f_{p}^{*}(x, y, z)$ to the true population $p d f$ $f_{p}(x, y, z)$. Let $\widehat{f}_{p}^{*}(x, y, z)$ be an estimate of $f_{p}^{*}(x, y, z)$ for a chosen $\theta_{Y Z}^{*}$. By [4], setting $t=(x, y, z)$ and $d t=d x d y d z$, the total estimation error can be decomposed as,

$$
\begin{aligned}
\int_{\mathbb{R}^{k}}\left|\widehat{f}_{p}^{*}(t)-f_{p}(t)\right| d t \leqslant & \int_{\mathbb{R}^{k}}\left|\widehat{f}_{p}^{*}(t)-f_{p}^{*}(t)\right| d t \\
& +\int_{\mathbb{R}^{k}}\left|f_{p}^{*}(t)-f_{p}(t)\right| d t
\end{aligned}
$$

where $k=\operatorname{dim}(x, y, z)$. The first term in the right hand side of (6.2) is the sampling error due to estimation of the other parameters in $\theta$, which can be estimated consistently from the samples $A$ and $B$. For given $\theta_{Y Z}^{*}$, the consistency of $\widehat{f}_{p}^{*}(x, y, z)$ guarantees that this term becomes negligible for large sample sizes $n_{A}$ and $n_{B}$. The second term represents the population model uncertainty or identification uncertainty, see in [9]. Obviously, the most favorable case, which occurs for instance if the CIA holds, is when the class $H_{p}$ consists of a single $p d f$. In this case, the population model for $(X, Y, Z)$ is identifiable and directly estimable from the available sample information.

Ignoring the informativness of the sampling designs introduces an implicit assumption 
that the population model holds for the sample data, in which case the class (6.1) is defined as the set of plausible distributions for $(X, Y, Z)$ having bivariate marginal $p d f \mathrm{~s} f_{A}(x, y), f_{B}(x, z)$, that is, the class $H_{p}\left[f_{A}(x, y), f_{B}(x, z)\right]$. Let $f_{A B}^{*}(x, y, z)$ be the population $p d f$ chosen from such a class and $\widehat{f}_{A B}^{*}(x, y, z)$ be an estimate of $f_{A B}^{*}(x, y, z)$. Similarly to (6.2), the total estimation error can be decomposed now as,

$$
\begin{aligned}
\int_{\mathbb{R}^{k}}\left|\widehat{f}_{A B}^{*}(t)-f_{p}(t)\right| d t \leqslant & \int_{\mathbb{R}^{k}}\left|\widehat{f}_{A B}^{*}(t)-f_{A B}^{*}(t)\right| d t+\int_{\mathbb{R}^{k}}\left|f_{A B}^{*}(t)-f_{p}^{*}(t)\right| d t \\
& +\int_{\mathbb{R}^{k}}\left|f_{p}^{*}(t)-f_{p}(t)\right| d t .
\end{aligned}
$$

Analogously to (6.2), the first term in the right hand side of (6.3) is the sampling error due to estimation. The second term represents now the matching error due to the informativness of sampling designs in $A$ and $B$. The last term is the population model uncertainty as defined in (6.2).

Remark 6. In [4] the notion of total estimation error is dealt in terms of cumulative distribution functions. In section 6 the total estimation error is defined in terms of density functions, so as to be consistent with what is done in previous sections where the effect of informative sampling on the sample pdf has been evaluated. Clearly, the use of density functions does not affect the interpretation of the results.

\section{Informative sampling from a trivariate normal population not satisfying the CIA}

In this section we analyze the effect of ignoring informative sampling designs for the case of a trivariate normal population distribution, but without imposing the CIA. Consider first the case of noninformative sampling. For this case, [8] shows that the only non-estimable unrestricted parameter is $\rho_{Y Z \mid X}$, the correlation between $Y$ and $Z$ given $X$. On the other hand, the unconditional correlation $\rho_{Y Z}$ is not unrestricted, because of the presence of the common variable $X$. Assuming that the $\mathrm{V}$-C matrix $\Sigma$ is positive 
semidefinite, the correlation $\rho_{Y Z}$ must be in the interval,

$$
\left[\tau_{p}, \nu_{p}\right]=\left[\rho_{Y X} \rho_{Z X}-A\left(\rho_{Y X}, \rho_{Z X}\right), \rho_{Y X} \rho_{Z X}+A\left(\rho_{Y X}, \rho_{Z X}\right)\right]
$$

where $A\left(\rho_{Y X}, \rho_{Z X}\right)=\sqrt{\left(1-\rho_{Y X}^{2}\right)\left(1-\rho_{Z X}^{2}\right)}$. All the values in the interval (7.1) are equally plausible for $\rho_{Y Z}$. Under the CIA, the parameter $\rho_{Y Z}$ is located at the midpoint of the interval (7.1), see [10]. The class $H_{p}$ of plausible population $p d f$ s for $(X, Y, Z)$ given by (6.1) is the set of three-variate normal distributions $f_{p}(x, y, z)$, with $\rho_{Y Z}$ in the interval (7.1). All the distributions in $H_{p}$ have the same mean vector $\mu$, and the $\mathrm{V}$-C matrices only differ in the entry of $\sigma_{Y Z}$ (or $\rho_{Y Z}$ in the correlation matrix). Each value of $\rho_{Y Z}$ in the interval (7.1) is associated with one, and only one, plausible population $p d f$ for $(X, Y, Z)$. Consequently, choosing a distribution from the class $H_{p}$ as a candidate for the actual joint $p d f$, is equivalent to choosing a value for $\rho_{Y Z}$ in the interval (7.1). The larger the class $H_{p}$, the more uncertain is the model for $(X, Y, Z)$. A simple and natural measure of the population model uncertainty, under complete marginal knowledge, is therefore $\left(\nu_{p}-\tau_{p}\right)$, (Eq. 7.1). The wider the interval, the more uncertain is $\rho_{Y Z}$ and hence, the greater is the uncertainty regarding $f_{p}(x, y, z)$. For further details on the uncertainty measures in parametric settings, see [17] and [6].

Next, consider the case of informative sampling. In this case, a set of maximum likelihood estimates for $\rho_{Y Z}$, (the likelihood ridge, as defined in [6]), can be evaluated. After computing the ML estimates of $\rho_{Y X}$ and $\rho_{Z X}$ as described in Section $3\left(\widehat{\rho}_{Y X}, \widehat{\rho}_{Z X}\right)$, the likelihood ridge for $\rho_{Y Z}$ in the informative case can be evaluated by substituting $\left(\widehat{\rho}_{Y X}, \widehat{\rho}_{Z X}\right)$ in the interval $(7.1)$.

Remark 7. When information regarding a statistical model for $(Y, Z)$ or $(Y, Z \mid X)$ is available, some models for $(X, Y, Z)$ might be excluded from the class (6.1), and the statistical model for $(X, Y, Z)$ becomes less uncertain, see [17], [5] and [3]. Under a parametric setting, such information assumes the form of constraints on the values of 
nonestimable parameters. For example, in the normal case, the information may consist of constraints on the values of the correlation $\rho_{Y Z}$ or equivalently, $\rho_{Y Z \mid X}$.

In what follows we study the effect of ignoring informative sampling designs on the class $H_{p}$ and the uncertainty measure $\left(\nu_{p}-\tau_{p}\right)$, under the sampling processes of Examples 1, 2 and 4, for the case of the trivariate normal population distribution. When the CIA does not hold, the reference sample model in the statistical matching context is given by

$$
f_{A \cup B}^{s m}\left(x_{i}, y_{i}, z_{i}\right)=f_{A \cup B}\left(x_{i}\right) f_{A}\left(y_{i} \mid x_{i}\right) f_{B}\left(z_{i} \mid x_{i}, y_{i}\right)
$$

Example 5. Under the expectations (5.2), the joint sample pdf is

$$
f_{S}\left(x_{i}, y_{i}, z_{i}\right)=f_{S}\left(x_{i}\right) f_{S}\left(y_{i} \mid x_{i}\right) f_{S}\left(z_{i} \mid x_{i}, y_{i}\right)
$$

where,

(a) $f_{S}\left(x_{i}\right)$ is $N\left(\mu_{X}+\left(\gamma_{X}+\gamma_{Z} \beta_{Z X \mid Y}+\left(\gamma_{Y}+\beta_{Z Y \mid X} \gamma_{Z}\right) \beta_{1}\right) \sigma_{X}^{2}, \sigma_{X}^{2}\right)$;

(b) $f_{S}\left(y_{i} \mid x_{i}\right)$ is $N\left(\mu_{Y \mid x_{i}}+\left(\gamma_{Y}+\gamma_{Z} \beta_{Z Y \mid X}\right) \sigma_{Y \mid X}^{2}, \sigma_{Y \mid X}^{2}\right)$;

(c) $f_{S}\left(z_{i} \mid x_{i}, y_{i}\right)$ is $N\left(\mu_{Z \mid x_{i} y_{i}}+\gamma_{Z} \sigma_{Z \mid X Y}^{2}, \sigma_{Z \mid X Y}^{2}\right)$;

(d) the joint sample pdf $f_{A \cup B}^{s m}\left(x_{i}, y_{i}, z_{i}\right)$ (7.2) is $N\left(\mu_{A \cup B}, \Sigma\right)$ with $\mu_{A \cup B}$ defined in the Supplementary Material, Equation (S6.6);

and $S=A, B$. The coefficient $\beta_{Z X \mid Y}\left(\beta_{Z Y \mid X}\right)$ is the partial regression coefficients of $Z$ on $X$ given $Y$ (the partial regression coefficients of $Z$ on $Y$ given $X$ ), $\sigma_{Z \mid X Y}^{2}$ is the residual variance in the regression of $Z$ on $X$ and $Y$. When $Y$ and $Z$ are conditionally independent given $X$, we are back to the results in Example 1. Ignoring the informative sampling designs implies in this case the assumption that the sample model coincides with the population model. Since $\Sigma_{A \cup B}=\Sigma\left(\Phi_{A \cup B}=\Phi\right.$ where $\Phi_{A \cup B}$ and $\Phi$ are the 
corresponding sample and population correlation matrices), the range of plausible values for $\rho_{Y Z}$, obtained under the added assumption that the sample correlation matrix is positive semidefinite, remains unchanged. In this case, ignoring the informative sampling designs affects the composition of the class $H_{p}$ since the mean vector changes from $\mu$ to $\mu_{A \cup B}$, but it does not affect the size of the class or the uncertainty measure $\left(\nu_{p}-\tau_{p}\right)$. See Supplementary Material, Section S6.

Example 6. Under the expectations (5.5), we obtain that

(a) $f_{S}\left(x_{i}\right)$ is $N\left(\mu_{X}+\left(\gamma_{X}+\gamma_{Y} \beta_{1}\right) \sigma_{X}^{2}, \sigma_{X}^{2}\right)$;

(b) $f_{S}\left(y_{i} \mid x_{i}\right)$ is $N\left(\frac{\mu_{Y \mid x_{i}}+\gamma_{Y} \sigma_{Y \mid X}^{2}}{1-2 \gamma_{2 Y} \sigma_{Y \mid X}^{2}}, \frac{\sigma_{Y \mid X}^{2}}{1-2 \gamma_{2 Y} \sigma_{Y \mid X}^{2}}\right)$;

(c) $f_{S}\left(z_{i} \mid x_{i}, y_{i}\right)$ is $N\left(\mu_{Z \mid x_{i}, y_{i}}, \sigma_{Z \mid X Y}^{2}\right)$;

(d) the joint sample pdf $f_{A \cup B}^{s m}\left(x_{i}, y_{i}, z_{i}\right)$ (7.2) is $N\left(\mu_{A \cup B}, \Sigma_{A \cup B}\right)$, with parameters defined in the Supplementary Material, Equations (S7.1), (S7.2);

where $S=A, B$. When $Y$ and $Z$ are conditionally independent given $X$, we are back to the results of Example 2. In this case, ignoring the informative sampling design affects both the class $H_{p}$ both the uncertainty measure. See Supplementary Material, Section S\%.

Example 7. Under the expectations (5.7), the sample pdf $f_{A \cup B}^{s m}\left(x_{i}, y_{i}, z_{i}\right)$ (7.2) is $N\left(\mu_{A \cup B}, \Sigma\right)$. Ignoring the informative sampling designs affects in this case the composition of the class $H_{p}$, but not the uncertainty measure $\left(\nu_{p}-\tau_{p}\right)$. See Supplementary Material, Section S8.

\section{Simulation study}

In order to illustrate the effects of ignoring the sampling process in statistical matching and to assess the performance of the imputation method described in Section 3, we performed a simulation study as described below.

\subsection{Simulation set-up}


The simulation experiment consists of the following four steps:

Step 1 Generate $N=2000$ independent population measurements $\left(x_{i}, y_{i}, z_{i}\right)$ from the following trivariate normal distribution satisfying the CIA:

$1.1 x_{i}$ is normal with parameters $\theta_{X}=\left(\mu_{X}, \sigma_{X}^{2}\right) ; \mu_{X}=5, \sigma_{X}^{2}=1$;

$1.2 y_{i} \mid x_{i}$ is normal with parameters $\theta_{Y \mid X}=\left(\beta_{0}+\beta_{1} x_{i} ; \sigma_{Y \mid X}^{2}\right) ; \beta_{0}=2, \beta_{1}=1$, $\sigma_{Y \mid X}^{2}=2$

$1.3 z_{i} \mid x_{i}$ is normal with parameters $\theta_{Z \mid X}=\left(\alpha_{0}+\alpha_{1} x_{i} ; \sigma_{Z \mid X}^{2}\right) ; \alpha_{0}=1, \alpha_{1}=0.5$, $\sigma_{Z \mid X}^{2}=2$.

Under the CIA, $\rho_{Y Z}=\rho_{Y X} \rho_{Z X}=0.19$.

Step 2 Draw independently samples $A$ and $B$ of size $n_{A}=n_{B}=400$ from the population generated in Step 1 by Poisson sampling, with selection probabilities

$$
\begin{aligned}
\pi_{i}^{A} & =n_{A} \frac{\exp \left(\gamma_{X}^{A} x_{i}+\gamma_{Y}^{A} y_{i}\right)}{\sum_{i=1}^{N} \exp \left(\gamma_{X}^{A} x_{i}+\gamma_{Y}^{A} y_{i}\right)} \\
\pi_{i}^{B} & =n_{B} \frac{\exp \left(\gamma_{X}^{B} x_{i}+\gamma_{Z}^{B} z_{i}\right)}{\sum_{i=1}^{N} \exp \left(\gamma_{X}^{B} x_{i}+\gamma_{Z}^{B} z_{i}\right)}
\end{aligned}
$$

We use different vectors $\gamma^{A}=\left(\gamma_{X}^{A}, \gamma_{Y}^{A}\right)$ and $\gamma^{B}=\left(\gamma_{X}^{B}, \gamma_{Z}^{B}\right)$, so as to distinguish between informative and non-informative samples. As shown in Example 1, these sampling probabilities preserve the CIA at the sample level. Notice that despite of the relatively large sampling fraction $f=(400 / 2000)$, for the sampling designs listed in Table 1 below, the percentage of common units in the samples $A$ and $B$ varies between $0.72 \%$ and $1.2 \%$.

Step 3 Construct a fused data set of size $\tilde{n}=N=2000$ in which the variables $(X, Y, Z)$ are jointly observed, as described in Section 3. For this, the population model parameters $\theta_{X}, \theta_{Y \mid X}, \theta_{Z \mid X}$ are estimated in three different ways: 
3.1 By ignoring the sample selection effects. Denote by $f_{1}\left(x_{i}, y_{i}, z_{i}\right)$ the estimated population distribution obtained in this case.

3.2 By assuming that the sampling probability coefficients $\gamma^{A}, \gamma^{B}$ are known and maximizing the sample likelihood (3.5) with respect to $\theta_{X}, \theta_{Y \mid X}, \theta_{Z \mid X}$. Denote by $f_{2}\left(x_{i}, y_{i}, z_{i}\right)$ the estimated population distribution obtained in this case.

3.3 By maximizing the observed sample likelihood (3.5) with respect to $\theta_{X}, \theta_{Y \mid X}, \theta_{Z \mid X}$, but where the expectations $E_{A}\left(w_{a}^{A} \mid x_{a}, y_{a} ; \gamma^{A}\right)$ and $E_{B}\left(w_{b}^{B} \mid x_{b}, z_{b} ; \gamma^{B}\right)$ appearing in the sample $p d f \mathrm{~s}$ are also estimated. First, the expectation $E_{A}\left(w_{a}^{A} \mid x_{a}, y_{a} ; \gamma^{A}\right)$ is estimated by a linear regression of $w_{a}^{A}$ on $\left(x_{a}, y_{a}\right)$. Second, $E_{A}\left(w_{a}^{A} \mid x_{a} ; \gamma^{A}\right)$ is evaluated as the integral of $E_{A}\left(w_{a}^{A} \mid x_{a}, y_{a} ; \gamma^{A}\right)$ with respect to the conditional sample $p d f f_{A}\left(y_{a} \mid x_{a}\right)$, and the integral is inserted into the likelihood, with the unknown sampling parameters $\gamma^{A}$ set at their estimated values, so that the likelihood is maximized with respect to the population parameters $\theta_{Y \mid X}$. A similar procedure is applied for estimating $E_{B}\left(w_{b}^{B} \mid x_{b}, z_{b} ; \gamma^{B}\right)$ and $\theta_{Z \mid X}$. Finally, in order to estimate the parameter $\theta_{X}$, the expectations $E_{A}\left(w_{a}^{A} ; \gamma^{A}\right)$ and $E_{B}\left(w_{b}^{B} ; \gamma^{B}\right)$ are expressed as the integrals of $E_{A}\left(w_{a}^{A} \mid x_{a} ; \gamma^{A}\right)$ and $E_{B}\left(w_{b}^{B} \mid x_{b} ; \gamma^{B}\right)$ with respect to the sample $p d f_{\mathrm{s}} f_{A}\left(x_{a}\right)$ and $f_{B}\left(x_{b}\right)$, with the corresponding sampling parameters set at their estimated parameters. Notice that by (8.1), the samples $A$ and $B$ are informative not only with respect to $Y$ and $Z$, but also with respect to $X$. Denote by $f_{3}\left(x_{i}, y_{i}, z_{i}\right)$ the estimated population distribution obtained in this case.

Step 4 Repeat Steps 2 and $3 M=500$ times for each choice of the coefficients $\gamma^{A}, \gamma^{B}$ defining the sample selection probabilities. We generated the population values only once (Step 1), so as to assess the design-based properties of the various estimation procedures. 


\subsection{Simulation results}

We start by studying the effect of ignoring the informative sampling mechanisms used for drawing the samples $A$ and $B$, when constructing the fused data set in Step 3. This is done by comparing the estimated population distributions $f_{1}\left(x_{i}, y_{i}, z_{i}\right)$ and $f_{2}\left(x_{i}, y_{i}, z_{i}\right)$. The results are shown in Tables 1-2 where the distance between the true marginal population $p d f \mathrm{~s}, f_{p}\left(x_{i}\right), f_{p}\left(y_{i}\right), f_{p}\left(z_{i}\right)$ and the corresponding estimated marginal $p d f_{\mathrm{s}} f_{h}\left(x_{i}\right), f_{h}\left(y_{i}\right), f_{h}\left(z_{i}\right)$, for $h=1,2$, are reported. In Table 3 , the distance between the true population $p d f, f_{p}\left(x_{i}, y_{i}, z_{i}\right)$, and the estimated $p d f_{\mathrm{s}} f_{h}\left(x_{i}, y_{i}, z_{i}\right)$, for $h=1,2,3$ is evaluated. As a measure of distance, we use the symmetric metric,

$$
K L_{p, h}\left(f_{p}, f_{h}\right)=0.5\left(K L_{p h}\left(f_{p}, f_{h}\right)+K L_{h p}\left(f_{h}, f_{p}\right)\right)
$$

where $K L_{p h}\left(f_{p}, f_{h}\right)$ is the Kullback-Leibler divergence between the two estimated $p d f_{\mathrm{s}} f_{p}$ and $f_{h}$. We computed for each of the 500 samples the metric (8.2) and then averaged the 500 values, which is viewed as the global divergence measure. Clearly, the smaller the average, the closer on average is the estimated population $p d f f_{h}$ to the true population pdf $f_{p}$, and the better should be the constructed data set in terms of mirroring the true data set. In Table 1 we report the KL-divergences (8.2) between the population $p d f f_{p}(x)$ and the estimated $p d f_{\mathrm{s}} f_{h}(x), h=1,2$. The predictive $p d f_{\mathrm{s}} f_{h}(x)$ are estimated from the sample data in $A, B$ and $A \cup B$. The corresponding KL-divergences are denoted as $K L_{p, h}^{X, A}, K L_{p, h}^{X, B}$ and $K L_{p, h}^{X, A \cup B}$. Although in pratice the $p d f f_{h}(x)$ would be estimated from the sample $A \cup B$, in Table 1 we also report the KL-divergences when the predictive $p d f_{\mathrm{S}} f_{h}(x) ; h=1,2$ are estimated based only on the data in $A$ or in $B$. This is done to illustrate how the informativness of the sampling designs acting in $A$ and $B$ combine in defining the sample model $f_{A \cup B}\left(x_{i}\right)$ and consequently, the KL-divergence $K L_{p, h}^{X, A \cup B}$, for $h=1,2$.

For $\gamma^{A}=(0,0) K L_{p, 1}^{X, A}$ coincides with $K L_{p, 2}^{X, A}$ since the sampling process acting in $A$ 
Table 1: $K L_{p, h}^{X, A}, K L_{p, h}^{X, B}, K L_{p, h}^{X, A \cup B}, \mathrm{~h}=1,2$, for different $\gamma^{A}, \gamma^{B}$ coefficients.

\begin{tabular}{|rrrrrrrr|}
\hline \multicolumn{1}{|c}{$\gamma^{A}$} & \multicolumn{1}{c}{$\gamma^{B}$} & $K L_{p, 1}^{X, A}$ & $K L_{p, 2}^{X, A}$ & $K L_{p, 1}^{X, B}$ & $K L_{p, 2}^{X, B}$ & $K L_{p, 1}^{X, A \cup B}$ & $K L_{p, 2}^{X, A \cup B}$ \\
\hline$(0.5,0)$ & $(0,0)$ & 0.125 & 0.002 & 0.002 & 0.002 & 0.031 & 0.001 \\
$(0,0)$ & $(0.5,0)$ & 0.002 & 0.002 & 0.126 & 0.002 & 0.031 & 0.001 \\
$(0,1)$ & $(0,0)$ & 0.257 & 0.021 & 0.002 & 0.002 & 0.056 & 0.003 \\
$(0,0)$ & $(0,1)$ & 0.002 & 0.002 & 0.091 & 0.010 & 0.020 & 0.003 \\
$(0.5,1)$ & $(0.5,1)$ & 0.500 & 0.055 & 0.288 & 0.037 & 0.379 & 0.043 \\
\hline
\end{tabular}

is not informative. When $\gamma^{A} \neq(0,0), K L_{p, 1}^{X, A}$ is always larger than $K L_{p, 2}^{X, A}$. The same is true for $K L_{p, 1}^{X, B}$ and $K L_{p, 2}^{X, B}$ when $\gamma^{B} \neq(0,0)$. Thus, ignoring the sample selection process affects negatively the quality of the predictions of $X$. Finally, since the sampling designs in $A$ and $B$ combine in defining the sample model $f_{A \cup B}\left(x_{i}\right), K L_{p, 1}^{X, A \cup B}$ is always between $K L_{p, 1}^{X, A}$ and $K L_{p, 1}^{X, B}$ and $K L_{p, 1}^{X, A \cup B}$ is always larger than $K L_{p, 2}^{X, A \cup B}$. Furthermore, the larger the informativness of the sampling processes, the larger is the distance between $f_{p}$ and $f_{h}$ in Table 1. Clearly, such a distance depends also on the characteristics of population $p d f$, that is, on the correlation structure between the variables of interest. For example, since $\rho_{Y X}=0.58>\rho_{Z X}=0.33$ ignoring the sampling processes with $\gamma^{A}=(0,1)$ and $\gamma^{B}=(0,0)$ yields $K L_{p, 1}^{X, A \cup B}=0.056$, which is larger than $K L_{p, 1}^{X, A \cup B}=0.020$ obtained when $\gamma^{A}=(0,0)$ and $\gamma^{B}=(0,1)$. In what follows, the predictive $p d f \mathrm{~s} f_{h}(x)$ are estimated from the sample $A \cup B$. Table 2 shows the KL-divergences, $K L_{p, h}^{Y}, K L_{p, h}^{Z}$, between the marginal population $p d f_{\mathrm{s}} f_{p}(y), f_{p}(z)$, and the corresponding estimated sample $p d f_{\mathrm{s}}$, $f_{h}(y), f_{h}(z) ; h=1,2$. As in Table $1, K L_{p, 1}^{Y}$ and $K L_{p, 1}^{Z}$ are always larger than $K L_{p, 2}^{Y}$ and $K L_{p, 2}^{Z}$, respectively. Thus, ignoring the sample selection process affects negatively the quality of the predictions of $Y$ and $Z$.

Table 2: $K L_{p, h}^{Y}, K L_{p, h}^{Z}, \mathrm{~h}=1,2$, for different $\gamma^{A}, \gamma^{B}$ coefficients.

\begin{tabular}{|rrrrrr|}
\hline \multicolumn{1}{|c}{$\gamma^{A}$} & \multicolumn{1}{c}{$\gamma^{B}$} & $K L_{p, 1}^{Y}$ & $K L_{p, 2}^{Y}$ & $K L_{p, 1}^{Z}$ & $K L_{p, 2}^{Z}$ \\
\hline$(0.5,0)$ & $(0,0)$ & 0.011 & 0.002 & 0.006 & 0.002 \\
$(0,0)$ & $(0.5,0)$ & 0.012 & 0.002 & 0.006 & 0.002 \\
$(0,1)$ & $(0,0)$ & 0.769 & 0.089 & 0.009 & 0.002 \\
$(0,0)$ & $(0,1)$ & 0.008 & 0.002 & 0.740 & 0.044 \\
$(0.5,1)$ & $(0.5,1)$ & 1.081 & 0.140 & 0.915 & 0.068 \\
\hline
\end{tabular}


In order to evaluate the performance of the imputation procedure proposed in Section 3, we computed the KL-divergence $K L_{p, h}^{X Y Z}$ between the true population $p d f f_{p}\left(x_{i}, y_{i}, z_{i}\right)$ and the estimated predictive models $f_{h}\left(x_{i}, y_{i}, z_{i}\right)$, for $h=1,2,3$. The results are presented in Table 3 for $\gamma^{B}=(0,0)$ and different $\gamma^{A}$ coefficients. The last column contains the mean sample size $M\left(n_{A}\right)$ over the $500 A$ samples selected in each case.

Table 3: $K L_{p, h}^{X Y Z}, \mathrm{~h}=1,2,3$ and mean sample size $M\left(n_{A}\right)$ over the 500 samples for different $\gamma^{A}$ coefficients.

\begin{tabular}{|rrrrr|}
\hline \multicolumn{1}{|c}{$\gamma^{A}$} & $K L_{p, 1}^{X Y Z}$ & $K L_{p, 2}^{X Y Z}$ & $K L_{p, 3}^{X Y Z}$ & $M\left(n_{A}\right)$ \\
\hline$(0.5,0)$ & 0.462 & 0.417 & 0.421 & 365 \\
$(0,1)$ & 1.272 & 0.509 & 0.530 & 379 \\
$(0.5,1)$ & 1.389 & 0.574 & 0.612 & 402 \\
\hline
\end{tabular}

As expected, ignoring the sample selection effects results in large KL measures. What we find encouraging is that the KL measures when estimating all the unknown parameters are not much larger than the corresponding measures when the sampling parameters are taken as known.

Tables 4 and 5 show the means and standard deviations (Sd) of the 500 estimates of the population parameters $\theta_{Y \mid X}=\left(\beta_{0}, \beta_{1}, \sigma_{Y \mid X}^{2}\right)$ under the predictive models $f_{h}\left(x_{i}, y_{i}, z_{i}\right)$; $h=1,3$. The tables help assessing the quality of the potential imputations obtained from the use of the two distributions. The means are denoted as $\overline{\widehat{\beta}}_{0, h}, \widehat{\widehat{\beta}}_{1, h}, \overline{\widehat{\sigma}}_{Y \mid X, h}^{2} ; h=1,3$.

Table 4: Means of estimates of $\left(\beta_{0}, \beta_{1}, \sigma_{Y \mid X}^{2}\right)$ over the 500 samples with different $\gamma^{A}$ coefficients. True parameters are $\beta_{0}=2, \beta_{1}=1, \sigma_{Y \mid X}^{2}=2$.

\begin{tabular}{|rrrrrrr|}
\hline \multicolumn{1}{|c}{$\gamma^{A}$} & $\overline{\widehat{\beta}}_{0,1}$ & $\overline{\widehat{\beta}}_{0,3}$ & $\overline{\widehat{\beta}}_{1,1}$ & $\overline{\widehat{\beta}}_{1,3}$ & $\overline{\widehat{\sigma}}_{Y \mid X, 1}^{2}$ & $\overline{\widehat{\sigma}}_{Y \mid X, 3}^{2}$ \\
\hline$(0.5,0)$ & 1.90 & 2.05 & 1.03 & 0.98 & 1.95 & 2.02 \\
$(0,1)$ & 5.08 & 2.10 & 0.69 & 0.97 & 1.29 & 1.99 \\
$(0.5,1)$ & 5.84 & 2.15 & 0.55 & 0.90 & 1.30 & 1.95 \\
\hline
\end{tabular}

As in Table 3, predictions based on $f_{1}\left(x_{i}, y_{i}, z_{i}\right)$ which ignores the sample selection effects produce a synthetic data set with distribution which differs from the true underlying population distribution. Consequently, subsequent inferential procedures 
Table 5: Standard deviations of estimates of $\left(\beta_{0}, \beta_{1}, \sigma_{Y \mid X}^{2}\right)$ over the 500 samples with different $\gamma^{A}$ coefficients.

\begin{tabular}{|rrrrrrr|}
\hline \multicolumn{1}{|c}{$\gamma^{A}$} & $s d\left(\widehat{\beta}_{0,1}\right)$ & $s d\left(\widehat{\beta}_{0,3}\right)$ & $s d\left(\widehat{\beta}_{1,1}\right)$ & $s d\left(\widehat{\beta}_{1,3}\right)$ & $s d\left(\widehat{\sigma}_{Y \mid X, 1}^{2}\right)$ & $s d\left(\widehat{\sigma}_{Y \mid X, 3}^{2}\right)$ \\
\hline$(0.5,0)$ & 0.32 & 0.37 & 0.06 & 0.06 & 0.04 & 0.04 \\
$(0,1)$ & 0.27 & 0.86 & 0.04 & 0.10 & 0.03 & 0.24 \\
$(0.5,1)$ & 0.30 & 1.23 & 0.05 & 0.14 & 0.03 & 0.36 \\
\hline
\end{tabular}

based on this data set will be subject to bias, even though the estimates obtained by ignoring the sample selection effects have the smallest variances, a well known phenomenon from other studies. For one of the 500 imputed data sets with $\gamma^{A}=(0.5,1)$, Figure 1 shows the population $p d f$, the kernel density estimate of the sample $p d f$, and the distribution of the imputed $Y$-values when using the predictive distribution $f_{3}\left(x_{i}, y_{i}, z_{i}\right)$. The bandwidth selection rule is as proposed in [21]. Similar results are obtained when using the average of the estimated parameters, reported in Table 4. As clearly seen, the sample $p d f$ is very different from the population $p d f$ due to the use of informative sampling, but the distribution of the imputed population values is close to the true population distribution.

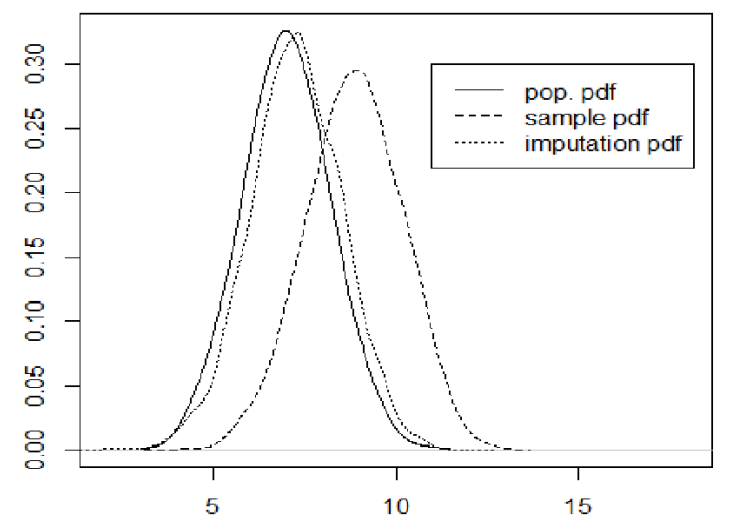

Figure 1: Population $p d f$, Kernel density estimate of the sample $p d f$ when ignoring the sample selection effects and distribution of the imputed $Y$-values.

Finally, Table 6 contains results of estimation of the correlation coefficient between $Y$ and 
$Z$ for the case where $\gamma^{B}=(0,0)$ and alternative sets of sampling coefficients $\gamma^{A}$. In order to evaluate the robustness of the procedure to deviations from the normality assumptions, we consider three different scenarios regarding the true population distribution:

Scenario 1 a three-variate normal distribution as in Step 1, with $\rho_{Y Z}^{(1)}=0.19$;

Scenario $2 x_{i}$ has a gamma distribution with shape 3 and scale $1 ; y_{i} \mid x_{i}$ and $z_{i} \mid x_{i}$ are normal with parameters as in Step 1, with $\rho_{Y Z}^{(2)}=0.39$;

Scenario $3 x_{i}$ has a gamma distribution with shape 3 and scale $1 ; y_{i} \mid x_{i}$ is normal as in Step $1 ; \log \left(z_{i} \mid x_{i}\right)$ is normal with parameters $\theta_{Z \mid X}=\left(\alpha_{0}+\alpha_{1} x_{i} ; \sigma_{Z \mid X}^{2}\right)$ with $\alpha_{0}=0.1$, $\alpha_{1}=0.4$ and $\sigma_{Z \mid X}^{2}=1$, with $\rho_{Y Z}^{(3)}=0.30$.

For each scenario 500 samples have been drawn from the corresponding population model and then 500 imputed data sets have been generated and used for estimating the correlation. In Table $6, \bar{\rho}_{Y Z, 1}^{(t)}$ and $\bar{\rho}_{Y Z, 3}^{(t)}, t=1,2,3$ represent the means of the correlation estimates from the 500 imputed data sets under the three scenarios, as obtained by use of the imputed values from the estimated predictive distributions $f_{1}\left(x_{i}, y_{i}, z_{i}\right)$ and $f_{3}\left(x_{i}, y_{i}, z_{i}\right)$.

Table 6: Correlation estimates under the three scenarios with different $\gamma^{A}$ coefficients. True correlations are $\rho_{Y Z}^{(1)}=0.19, \rho_{Y Z}^{(2)}=0.39, \rho_{Y Z}^{(3)}=0.30$.

\begin{tabular}{|rrrrrrr|}
\hline \multicolumn{1}{|c}{$\gamma^{A}$} & $\bar{\rho}_{Y Z, 1}^{(1)}$ & $\bar{\rho}_{Y Z, 3}^{(1)}$ & $\bar{\rho}_{Y Z, 1}^{(2)}$ & $\bar{\rho}_{Y Z, 3}^{(2)}$ & $\bar{\rho}_{Y Z, 1}^{(3)}$ & $\bar{\rho}_{Y Z, 3}^{(3)}$ \\
\hline$(0.5,0)$ & 0.21 & 0.19 & 0.48 & 0.39 & 0.49 & 0.36 \\
$(0,1)$ & 0.18 & 0.19 & 0.47 & 0.39 & 0.50 & 0.39 \\
$(0.5,1)$ & 0.16 & 0.18 & 0.46 & 0.38 & 0.46 & 0.35 \\
\hline
\end{tabular}

Under the first scenario, the means of the estimated correlation coefficients based on the predictive distributions $f_{1}\left(x_{i}, y_{i}, z_{i}\right)$ and $f_{3}\left(x_{i}, y_{i}, z_{i}\right)$ are similar with small empirical bias. However, under the second and third scenarios, the mean estimates when ignoring the sample selection effects show large empirical bias, where as the estimates obtained when accounting for the sampling effects reveal only small bias. 


\section{Summary}

In this paper we analyzed the statistical matching problem when the two sampling processes used to select the samples $A$ and $B$ are informative. The conditions guaranteeing the identifiability and estimability of the sample likelihood are investigated. When the CIA does not hold, an uncertainty analysis is carried out, showing how ignoring the sampling selection can affect the matching error. Furthermore, the effect of alternative informative sampling designs on the inference process for a trivariate normal population is studied. Finally, a simulation experiment has been performed showing that the magnitude of the error will depend on both the informativeness of the sampling processes and the correlations between the variables of interest. As our paper shows, accounting for the sampling designs effects improves the quality of the matched data file very significantly. The proposed procedure based on maximization of the sample likelihood reveals good performance in terms of the Kullback-Leibler divergence and population parameters estimation and hence in enabling good estimation of the population distribution and imputation of missing observations. Clearly, more theoretical and empirical studies with different population distributions and sampling designs are needed to further ascertain the results of the present paper.

Supplementary Materials The Supplementary Material contains the proof of Proposition 1 and of the results in Examples 1-7.

Acknowledgment The authors are grateful to the referee for very careful reading of the manuscript and thoughtful comments. We dedicate this paper to the memory of Professor Susanne Rässler, who passed away last year while still young and very active in the area of statistical matching. 


\section{References}

[1] Conti, P.L., Marella, D. and Scanu, M. (2012) Uncertainty Analysis in Statistical Matching. Journal of Official Statistics, 28, pp.1-21.

[2] Conti, P.L., Marella, D. and Scanu, M. (2013) Uncertainty Analysis for Statistical Matching of ordered categorical variables. Computational Statistics \& Data Analysis, 68, pp.311-325.

[3] Conti, P.L., Marella, D. and Scanu, M. (2015) How far from identifiability? A systematic overview of the statistical matching problem in a non-parametric framework. Communications in Statistics - Theory and Methods, 1, 2, pp.967-994.

[4] Conti, P.L., Marella, D. and Scanu, M. (2016) Statistical matching analysis for complex survey data with applications. Journal of the American Statistical Association, 111, 516, pp.1715-1725.

[5] D’Orazio, M., Di Zio, M. and Scanu, M. (2006a). Statistical Matching for Categorical Data: Displaying Uncertainty and Using Logical Constraints. Journal of Official Statistics, 22, pp.137-157.

[6] D’Orazio, M., Di Zio, M. and Scanu, M. (2006b). Statistical Matching: Theory and Practice. Chichester: Wiley.

[7] Feder, M. and Pfeffermann, D. (2018). Statistical Inference Under Non-ignorable Sampling and Non-response-An Empirical Likelihood Approach. Paper available from the authors.

[8] Kadane, J.B., (2001). Some statistical problems in merging data files. Journal of Official Statistics, 17, pp.423-433.

[9] Manski, C. F. (2003). Partial Identification of Probability Distributions. New York: Springer-Verlag. 
[10] Moriarity, C. and Scheuren, F., (2001). Statistical Matching: A Paradigm of Assessing the Uncertainty in the Procedure. Journal of Official Statistics, 17, pp.407422.

[11] Okner, B. (1972). Constructing a new data base from existing microdata sets: the 1966 merge file. Annals of Economic and Social Measurement, 1, pp.325-342.

[12] Pfeffermann, D., Krieger, A.M. and Rinott, Y. (1998). Parametric distribution of complex survey data under informative probability sampling. Statistica Sinica, 8, pp.1087-1114.

[13] Pfeffermann, D. and Sverchkov, M. (1999). Parametric and semi-parametric estimation of regression models fitted to survey data. Sankhyā : The Indian Jounal of Statistics 61, pp.166-186.

[14] Pfeffermann, D. and Sverchkov, M. (2009). Inference under Informative Sampling. In: Handbook of Statistics 29B; Sample Surveys: Inference and Analysis. Eds. D. Pfeffermann and C.R. Rao. North Holland.

[15] Pfeffermann, D. (2011). Modelling of complex survey data: Why model? Why is it a problem? How can we approach it? Survey Methodology, 37, 2, pp.115-136.

[16] Pfeffermann, D., and Landsman, V. (2011). Are private schools really better than public schools? Assessment by methods for observational studies. Annals of Applied Statistics, 5, pp.1726-1751.

[17] Rässler, S.(2002). Statistical Matching: A Frequentist Theory, Practical Applications and Alternative Bayesian Approaches. New York: Springer.

[18] Renssen, R. (1998). Use of statistical matching techniques in calibration estimation. Survey Methodology, 24, pp.171-183. 
[19] Rodgers, W.L. (1984). An Evaluation of Statistical Matching. Journal of Business and Economic Statistics, 1, pp.91-102.

[20] Rubin, D.B. (1986). Statistical Matching using file concatenation with adjusted weights and multiple imputations. Journal of Business and Economics Statistics, 4, pp.87-94.

[21] Sheather, S.J. and Jones, M.C. (1991). A Reliable Data-Based Bandwidth Selection Method for Kernel Density Estimation. Journal of the Royal Statistical Society Series $B, \mathbf{5 3}$, pp.683-690.

[22] Sims, C. A. (1972). Comments on: "Constructing a New Data Base From Existing Microdata Sets: the 1966 Merge File", by B.A. Okner. Annals of Economic and Social Measurements, 1, pp.343-345.

[23] Singh, A.C., Mantel, H., Kinack, M. and Rowe, G., (1993). Statistical Matching: Use of Auxiliary Information as an Alternative to the Conditional Independence Assumption. Survey Methodology, 19, pp.59-79.

[24] Skinner, C.J., Holt, D. and Smith, M.F. (1989). Analysis of complex surveys. Wiley. 